\title{
Optical Frequency Up-Conversion of UWB Monocycle Pulse Based on Pulsed-Pump Fiber Optical Parametric Amplifier
}

\author{
Jia Li, Yu Liang, Xing Xu, Kim K. Y. Cheung, and Kenneth K. Y. Wong* \\ Photonic Systems Research Laboratory, \\ Department of Electrical and Electronic Engineering, \\ The University of Hong Kong \\ *Email: kywong@eee.hku.hk
}

\begin{abstract}
We propose a method to realize frequency up-conversion of UWB monocycle pulse using pulsed-pump fiber optical parametric amplifier (OPA). The spectrum of the amplified signal contains many discrete frequency components which are separated by the modulation frequency of the pump. Each frequency components contain the same spectral information as that of the original signal. By selecting the first-order or higher-order frequency components of the amplified signal and beating in the photodetector, up-converted signal at different frequencies are obtained. We demonstrate frequency up-conversion of baseband UWB monocycle pulse from 3-GHz to $19-\mathrm{GHz}$ in the experiment and frequency up-conversion of pseudo-random binary sequence (PRBS) signal from 3-GHz to $60-\mathrm{GHz}$ in the simulation.
\end{abstract}

Keywords: frequency up-conversion, microwave photonics, optical parametric amplifier, ultra-wideband

\section{INTRODUCTION}

Ultra-wideband (UWB) technology has been widely investigated recently due to its intrinsic properties to overcome the scarcity of available radio-frequency resource. However, the ultra-low emission power limits its transmission distance to tens of meters. Therefore, UWB over fiber is proposed by utilizing low-loss optical fiber as the transmission medium so as to extend the coverage area. Many methods have been proposed to generate baseband UWB signals $(7.5 \mathrm{GHz}) \mathrm{using}$ photonic methods ${ }^{1-5}$. Besides signal generation function, frequency up-conversion function also plays an important role in either UWB over fiber, or Radio over fiber (RoF) systems. The driving force for implementing microwave or millimeter-wave (MMW) over fiber comes from the ever increasing demand for high-data-rate wireless communications. And microwave or MMW communication is attractive for its large bandwidth and spectrum availability. To generate microwave or MMW signals, frequency up-conversion is needed where a baseband signal is mixed with a high frequency local oscillator (LO). However, frequency up-conversion in the high frequency regime is difficult to be realized in the electrical domain due to the bottleneck of electronics, so it is better to perform frequency up-conversion function in the optical domain. Moreover, it is cost effective to centralize the broadband mixing in the center office instead of each base station.

In the past few years, researchers have extensively investigated the generation of MMW signals using photonic methods. Traditionally there are three ways to generate MMW signals: direct intensity modulation ${ }^{6}$, external modulation ${ }^{7}$ and remote heterodyning ${ }^{8}$. However these methods are practically not useful, since for direct modulation it is limited by the modulation bandwidth of the laser diodes which is difficult for MMW signal generation, for external modulation it needs high frequency and high cost electrical driving source and it has low tolerance to dispersion when transmitting in the fiber, for heterodyning method it needs a precisely biased and very stable electrooptic modulator or complex laser systems to lock the phase relationship between the two lasers. Recently, several other approaches for frequency upconversion has been proposed such as four-wave mixing (FWM) in the highly-nonlinear dispersion-shifted fiber (HNL$\mathrm{DSF})^{9}$, cross-phase modulation (XPM) in the semiconductor optical amplifier (SOA) ${ }^{10}$, cross-gain modulation (XGM) in $\mathrm{SOA}^{11}$, and external phase modulator ${ }^{12}$.

Although many approaches have been proposed and demonstrate for microwave or MMW signal up-conversion, few researchers have demonstrated UWB signal up-conversion to $24 \mathrm{GHz}$ or $60 \mathrm{GHz}$. One method is based on selfheterodyne technique using an arrayed waveguide grating $(\mathrm{AWG})^{13}$, which requires special MZM with high extinction

Optical Transmission Systems, Switching, and Subsystems VII, edited by Dominique Chiaroni,

Proc. of SPIE-OSA-IEEE Asia Communications and Photonics, SPIE Vol. 7632, 76322C

(c) 2009 SPIE-OSA-IEEE • CCC code: 0277-786X/09/\$18・ doi: 10.1117/12.852086

SPIE-OSA-IEEE/ Vol. 7632 76322C-1 
ratio to suppress residual carrier to meet the UWB emission mask. However this method has a good tolerance to fiber dispersion. Another method is based on direct modulation in a MZM in the nonlinear regime ${ }^{14}$, which is simple and compact. Nevertheless it needs expensive high-speed electronic devices which make it difficult to be upgraded to a 60$\mathrm{GHz}$ frequency up-converter. A method based on nonlinear polarization rotation in a SOA is also demonstrated with a broad conversion range of the whole $C$-ban ${ }^{15}$. But it suffers from the slow carrier recovery speed which limits its performance in the high frequency regime. Furthermore, MMW UWB signal after fiber transmission is analyzed $\mathrm{in}^{16}$, which shows the doublet pulse has better tolerance to fiber dispersion than the monocycle pulse.

A novel fiber OPA-based MMW UWB signal generation has been proposed and demonstrated via frequency upconversion method. The baseband UWB signal is amplified by a high-repetition-rate pulsed pump and generates many sidebands separated by the modulation frequency of the pump. By selecting two or three of the sidebands and beating in the photodetector, we can obtain an up-converted signal in the MMW band. This method has the advantages of large mixing bandwidth due to the ultrafast response property in the optical fiber. In addition, this method can be upgraded into a $60-\mathrm{GHz}$ up-converter by selecting the higher order sidebands without using high-frequency electrical components. Furthermore, it can be deployed in the remote local oscillator delivery scheme to mitigate the dispersion effect in the long reach access networks and serves as both frequency up-converter and signal amplifier in a local exchange ${ }^{17}$ because of its all-optical operation and positive optical gain. In the experiment, UWB signal has been up-converted from 3 to $\sim 19$ GHz with 18-dB optical gain using fiber OPA.

\section{OPERATING PRINCIPLE}

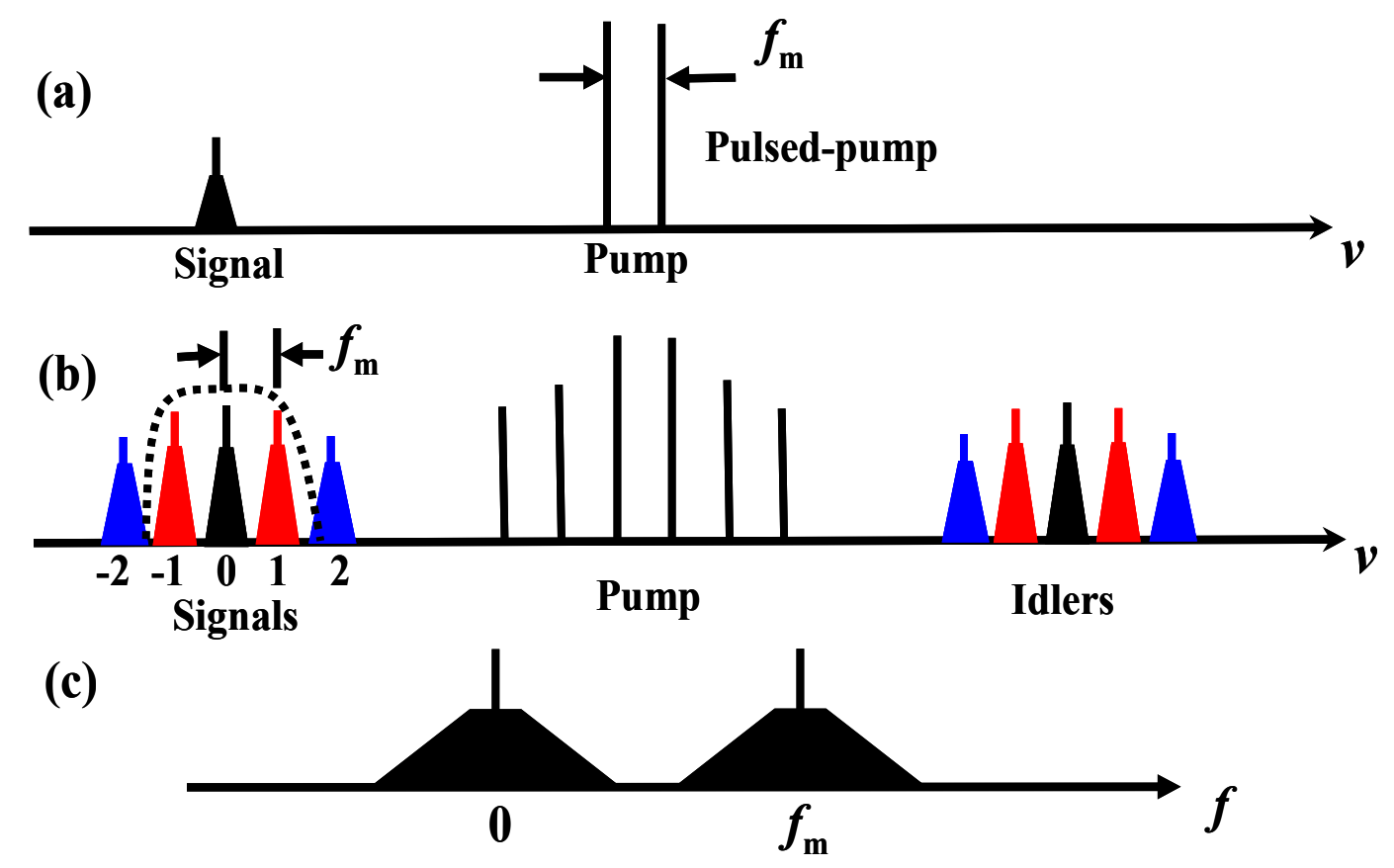

Fig.1. Principle of operation. (a) Input spectrum. (b) Output spectrum. (c) Filtered output electrical spectrum.

The principle of UWB frequency up-converter is shown in Fig. 1. The pump consists of two narrowly spaced equalpower continuous monochromatic waves with a frequency separation of $f_{\mathrm{m}}$, which can equally be viewed as a single wave with average frequency $v_{\mathrm{c}}$ and a cosine-squared modulation at the beat frequency $f_{\mathrm{m}}$. Then the high power pump is co-propagating with a weak signal along a spool of nonlinear fiber. After parametric amplification, the signal spectrum at the output of the nonlinear fiber will consist of multiple discrete frequency peaks separated by a frequency of $f_{\mathrm{m}}$, and each frequency peak contains the replica of the input signal spectrum as shown in Fig. 1(b). It is the same for the idlers generated at the other side of the pump except that the spectrum of each idler is inverted with respect to that of the 
original signal. The newly generated frequency peaks beside the original two pump waves are due to the self-phase modulation (SPM) effect. According to a quasi-continuous wave (CW) calculation the output signal spectrum can be written as ${ }^{18}$ :

$$
B_{s}(L, f)=\sum_{n=-\infty}^{\infty} a_{n} B_{s}\left(0, f-n f_{m}\right)
$$

where $B_{\mathrm{s}}(0, f)$ is the input signal spectra, $B_{\mathrm{s}}(L, f)$ is the output signal spectra and $a_{n}$ is conversion coefficients for each newly generated sidebands. As the sidebands are inherited from parametric gain from the pump, they are phasecorrelated with each other. Therefore, we can select two or three neighboring frequency components by using a narrowband optical filter and launch them into the photodetector (PD). After beating between the sidebands, a stable MMW signal at frequency $f_{\mathrm{m}}$ will be generated and the spectrum of the original signal will also be up-converted to this frequency as shown in Fig. 1(c).

Furthermore, in order to upgrade this system to $60-\mathrm{GHz}$ frequency it is not necessary to increase the electrical source frequency and choose high-speed electrooptic modulator as the other methods showed. It can be achieved by simply selecting two second-order sidebands $(n=-2,2)$ as shown in Fig. 1 (b) of the output signal spectrum and beating in the $\mathrm{PD}$. The frequency of the electrical source required in this approach is only $7.5 \mathrm{GHz}$ which significantly reduce the system cost and complexity. This method can also be used for MMW signal generation and frequency up-conversion for other types of baseband signal.

\section{EXPERIMENTAL SETUP}

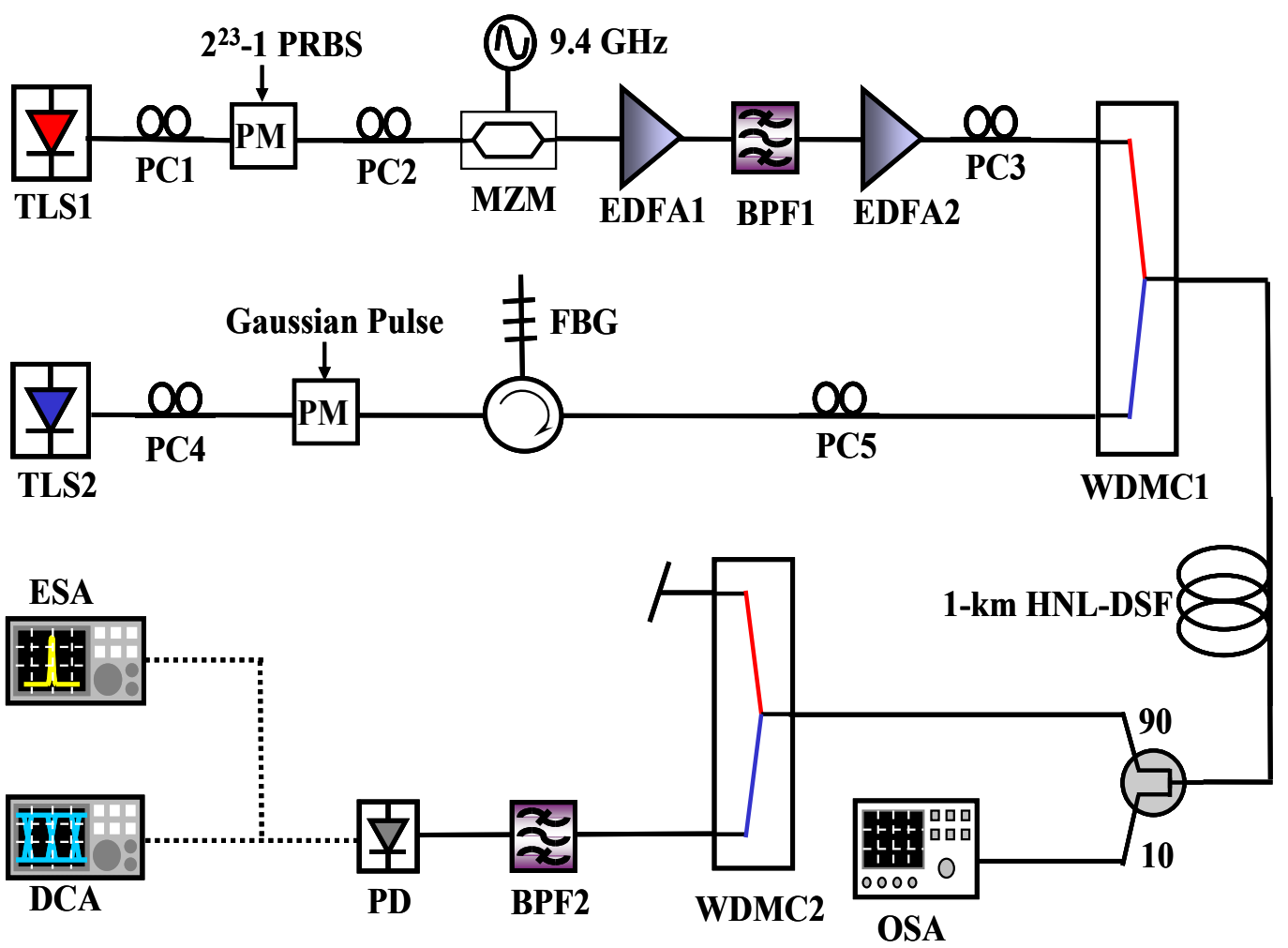

Fig. 2. Experimental setup for MMW UWB frequency up-conversion using fiber OPA. Refer to text for detailed description. 
Fig. 2 shows the experimental setup for MMW UWB monocycle and doublet pulses generation and up-conversion. A tunable laser source (TLS) TLS2 was used to generate a CW signal. It was then phase modulated by a PM with a train of Gaussian pulses with a duty ratio of $1 / 20$ at a bit rate of $9.4 \mathrm{~Gb} / \mathrm{s}$. So the repetition rate of the generated pulse was 470 $\mathrm{Mb} / \mathrm{s}$. Then the phase modulated signal was launched into a circulator and a FBG to achieve phase modulation to intensity modulation conversion by using the fiber Bragg grating (FBG) as a frequency discriminator ${ }^{4}$. The baseband UWB monocycle and doublet pulse could be generated by locating the phase-modulated optical carrier at the linear or quadrature slope of the transmission profile of the FBG. In our experiment, the center wavelength of the FBG was located at $1557.701 \mathrm{~nm}$ with a 3-dB bandwidth of $0.20 \mathrm{~nm}$, the wavelength of the signal was chosen to be $1557.596 \mathrm{~nm}$ to generate baseband UWB monocycle pulse. Since the output power of the UWB signal was around $-10.2 \mathrm{dBm}$ which was too small for detection, we used another erbium-doped fiber amplifier (EDFA) to amplify it before characterizing its temporal and spectral performance. Meanwhile, TLS1 was served as the pump with a wavelength of $1543 \mathrm{~nm}$. It was phase modulated by a 10-Gb/s $2^{23}-1$ PRBS to suppress stimulated Brillouin scattering (SBS). Then the pump wave was coupled into a Mach-Zehnder modulator (MZM) biased at the transmission null to act as a double sideband with optical carrier suppressed (DSB-OCS) modulation. The MZM was driven by an electrical sine wave with a frequency of 9.4 $\mathrm{GHz}$ to generate two pump waves separated by $18.8 \mathrm{GHz}$. The frequency chosen as $18.8 \mathrm{GHz}$ instead of $24 \mathrm{GHz}$ was mainly limited by the ESA used which has a range up to $26 \mathrm{GHz}$. However this method also works for 24-GHz upconversion case due to the ultra-fast response time in the optical fiber. Then the pump was amplified by a two-stage EDFA (EDFA1\&2) to a power of $25.44 \mathrm{dBm}$. A tunable bandpass filter (TBPF) TBPF1 was used to suppress amplified spontaneous noise (ASE) noise coming from the EDFAs. The pump and signal waves were coupled together by a wavelength-division multiplexing coupler (WDMC) WDMC1 and launched into 1-km HNL-DSF with zero-dispersion wavelength of $1542 \mathrm{~nm}$ and nonlinear coefficient of $10.4 \mathrm{~W}^{-1} \mathrm{~km}^{-1}$. Polarization controllers (PC3 \& PC5) were located in the signal and pump branches to align the SOP of the two waves to maximize OPA gain. The optical spectrum after the HNL-DSF was analyzed in the OSA. Then the pump wave was discarded using another WDMC2 and the up-converted signal was further filtered by a narrow-band BPF2 to select the three frequency peaks in the center of the spectrum. If we select only two of them, the electrical power generated would be rather low for detection. Then the three waves are injected into a PD, and finally monitored in the DCA and the ESA.

\section{RESULTS AND DISCUSSIONS}
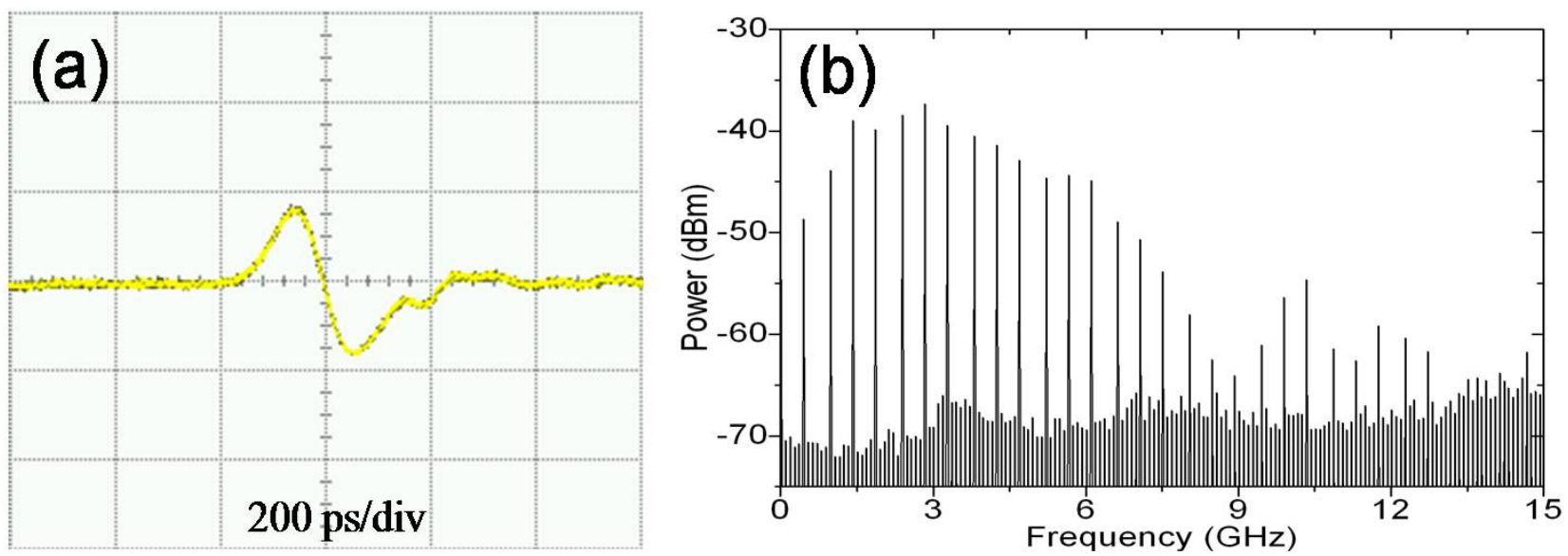

Fig. 3. (a) Waveform of generated baseband monocycle pulse. (b) Corresponding power spectrum of the monocycle pulse.

First of all, baseband UWB pulse was generated through PM-IM conversion method. The waveforms and the corresponding power spectra are shown in Fig. 3. It can be observed that the monocycle pulse had a center frequency of $2.82 \mathrm{GHz}$ and a $-10-\mathrm{dB}$ bandwidth of $5.82 \mathrm{GHz}$, while the doublet pulse had a center frequency of $4.69 \mathrm{GHz}$ and a -10 $\mathrm{dB}$ bandwidth of $4.82 \mathrm{GHz}$. The discrepancy between the experimental result and the FCC mask for baseband UWB was 
because of the non-ideal transmission profile of the FBG used, which should have a steep rising or falling slope and good linearity of the slope as well. Since the slope was not steep enough in the experiment, the generated pulse had a wide pulse shape which results in a low center frequency and narrow bandwidth. The non-ideal linearity of the slope leads to the fluctuation in amplitude and nonsymmetry in the pulse shape. So in order to generate FCC-compliant baseband UWB signal specially designed FBG is required.

Fig. 4 (a) shows the optical spectrum after the HNL-DSF in the OSA. It can be observed that an on-off OPA gain of 18 $\mathrm{dB}$ has been achieved in the experiment which shows re-amplification capability of the original signal. And the spectrum of the amplified signal, idler and the pump showed many frequency peaks due to the SPM and pulsed-pump OPA gain as mentioned in the principle section. Fig. 4 (b) shows the spectrum of the output signal in a larger scale with a resolution of $0.01 \mathrm{~nm}$. It is clearly seen that many sidebands which contain the replica of the original signal spectrum were generated around the original signal spectrum with a frequency separation of $18.8 \mathrm{GHz}$ just as the theory predict. However, the frequency spacing between the higher-order sidebands was $9.4 \mathrm{GHz}$ instead of $18.8 \mathrm{GHz}$, which was because of the low carrier suppression ration of the pump that leaded to the residual $9.4 \mathrm{GHz}$ component in the pump wave. As a solution, a dual-arm MZM could be used which was biased at the minimum transmission point and driven by two complementary 9.4-GHz sinusoidal waveforms 9 .

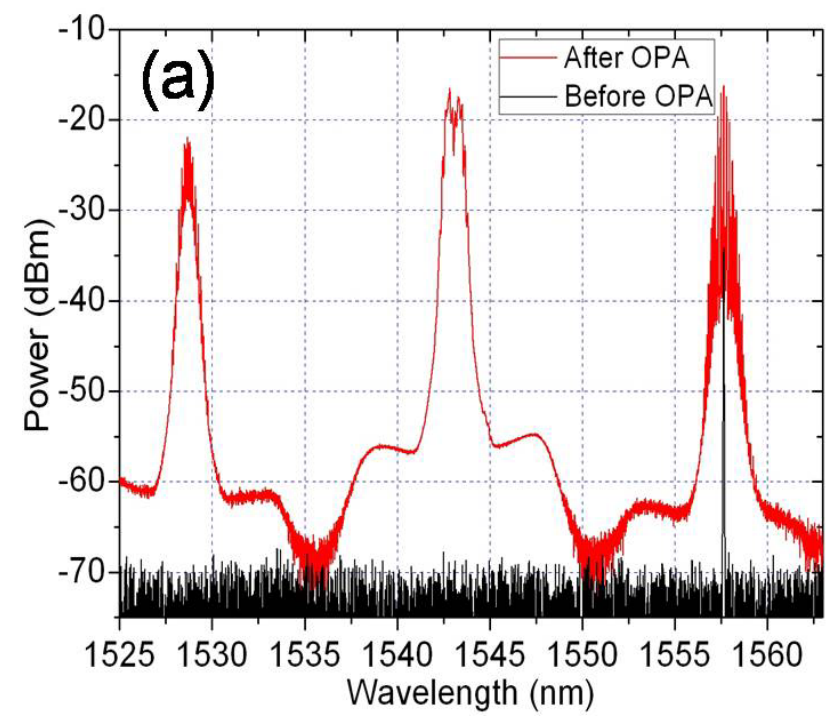

Fig. 4. (a) Optical spectrum before and after OPA.

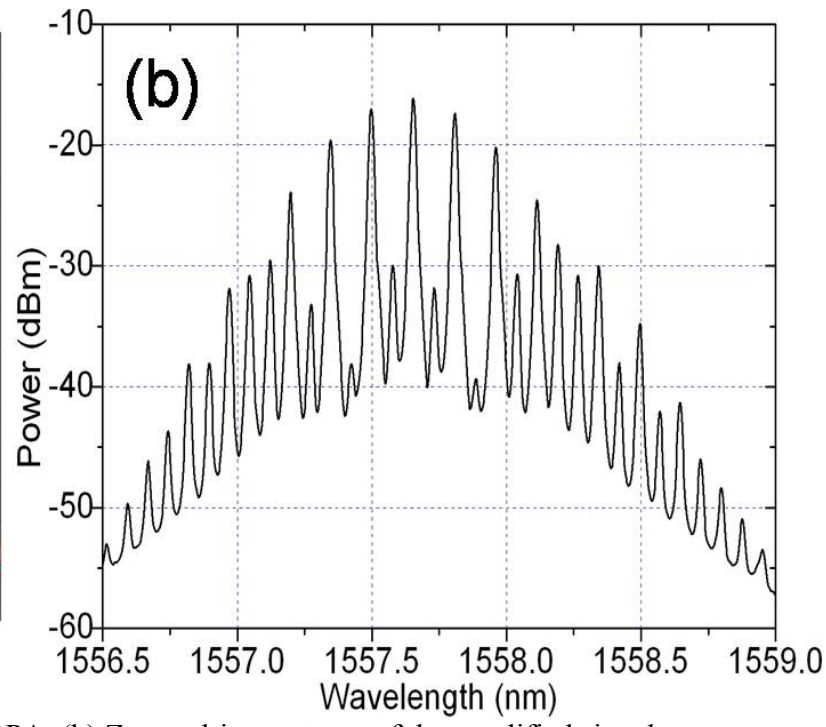

(b) Zoomed-in spectrum of the amplified signal.
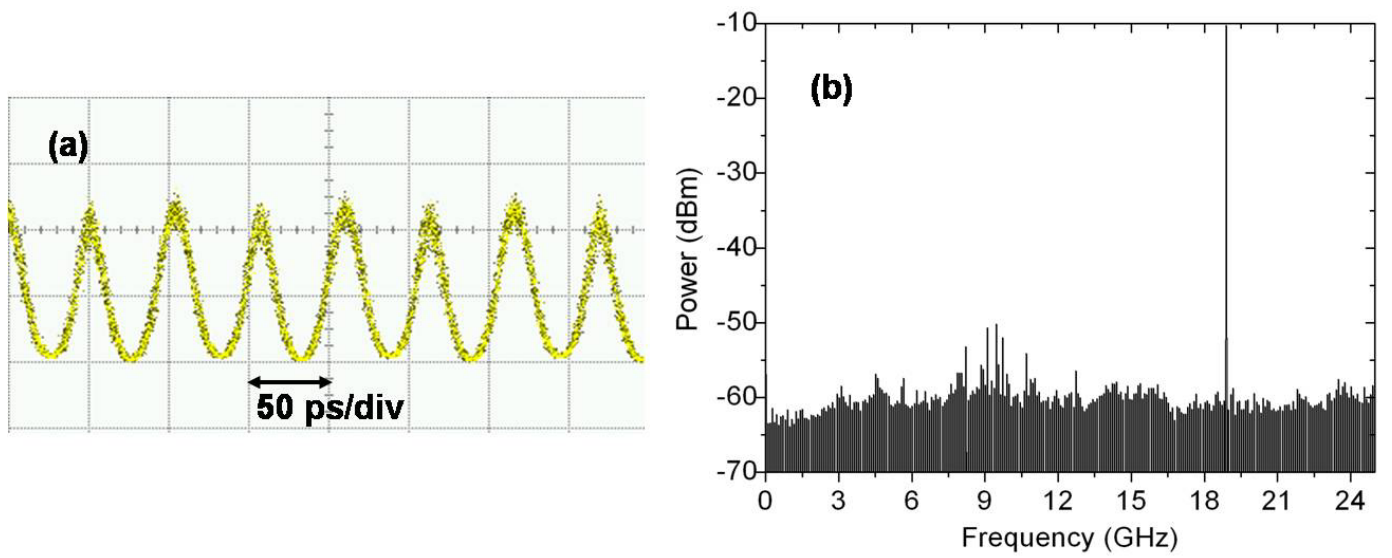

Fig. 5. (a) Waveform of the generated MMW signal at $18.8 \mathrm{GHz}$. (b) Corresponding power spectrum of the MMW signal. 
As a MMW signal generator, no signal was fed into the PM and a BPF was used to select the three main frequency components in the spectrum as shown in Fig. 5. After beating in the PD, MMW signal at frequency $18.8 \mathrm{GHz}$ was generated as shown in Fig. 5 (a). The corresponding electrical spectrum was shown in Fig. 5 (b). It can be observed that residual RF component still exists in $9.4 \mathrm{GHz}$ due to the non-ideal suppression of the optical carrier in the pump wave. However a main-to-sidelobe ratio (MSR) of over $40 \mathrm{~dB}$ has been obtained in the RF spectrum.

For MMW UWB signal generation, the PM was driven by the periodical Gaussian pulse train. Fig. 6 (a) shows the waveform of the up-converted UWB monocycle pulse. It can be observed that the carrier was a pulse train with a frequency of $18.8 \mathrm{GHz}$ and the envelopes resembled the original baseband UWB signal as shown in the waveform with red dashed lines. The corresponding power spectra of the up-converted UWB signal is shown in Fig. 6 (b). It can be seen that both baseband and MMW UWB signals were obtained in the spectrum just as the theory predict. The MMW monocycle pulse had a center frequency of $21.73 \mathrm{GHz}$ and a $-10-\mathrm{dB}$ bandwidth of $5.76 \mathrm{GHz}$. It is clearly observed that the bandwidth is almost maintained during the up-conversion process. A strong frequency component also appeared at $18.8 \mathrm{GHz}$ in the spectrum due to the beating between the optical carriers, which could be mitigated by carrier suppression using FBGs. Electrical UWB filters located at $24-\mathrm{GHz}$ band should be used to eliminate undesirable lowfrequency or baseband components to avoid interference with other wideband services before radiation. As an efficient way, the baseband spectral lines can be reused for the baseband UWB communication. Although up-converted signals were not located in the frequency range from $22 \mathrm{GHz}$ to $29 \mathrm{GHz}$, it can be extended into a 24-GHz UWB signal generator by using a higher modulation frequency. Furthermore, we can also extend this method into a $60-\mathrm{GHz}$ upconversion case by beating the two second-order sidebands in the PD using a modulation frequency of only $7.5 \mathrm{GHz}$.
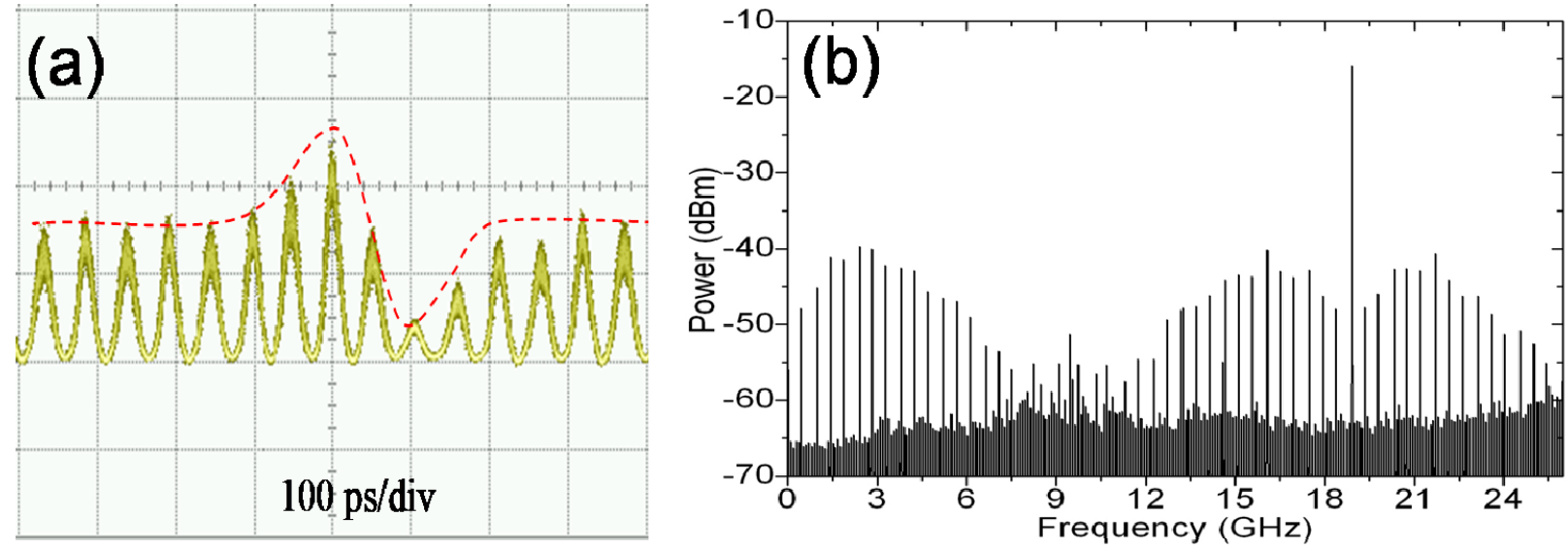

Fig. 6. (a) Waveform of the up-converted UWB monocycle pulse. (b) Corresponding power spectrum of the up-converted UWB monocycle pulse.

In order to verify our method, simulation has been done for a $60-\mathrm{GHz}$ frequency up-conversion of baseband PRBS signal. The simulation setup is shown in Fig. 7. The simulation tool was RSoft Photonics CAD Suite Optsim. The pump wavelength was set to be $1543 \mathrm{~nm}$ and the power is $24.8 \mathrm{dBm}$. The signal wavelength was set to be $1560 \mathrm{~nm}$ and the input power was $-10 \mathrm{dBm}$. Zero-dispersion wavelength of the HNL-DSF was $1542 \mathrm{~nm}$ and the nonlinear coefficient was $10.4 \mathrm{~W}^{-1} \mathrm{~km}^{-1}$. The fiber length was $1 \mathrm{~km}$. The OCs in the simulation was lossless, and the fiber loss was set to be 0.2 $\mathrm{dB} / \mathrm{km}$. The pump wave TLS1 was intensity modulated by a sine wave with a frequency of $7.5-\mathrm{GHz}$. And the signal wave TLS2 was IM by a 3-Gb/s $2^{7}-1$ PRBS pattern. After the parametric amplification in the HNL-DSF, many sidebands were generated besides the original signal spectrum as shown in Fig. 7. And two second-order sidebands were selected using two BPFs (BPF2 \&BPF3) with 3-dB bandwidth $0.16 \mathrm{~nm}$. Then they were combined together and the spectrum is shown in Fig. 8. After photodetection, the baseband signal was up-converted to 60-GHz band as shown in Fig. 9 (a,b). After that, it was filtered by an electrical BPF1 with a center frequency of $60 \mathrm{GHz}$ and mixed with a $60-\mathrm{GHz}$ sine wave in the mixer. A phase shifter was used to align the phase of the $60-\mathrm{GHz}$ source with the up-converted signal. After downconversion in the mixer the signal was filtered by another electrical BPF2 and monitored in the DCA and ESA as shown 
in Fig. 9 (c,d). From the waveforms and spectrum it could be seen that a 60-GHz frequency up-conversion was successfully demonstrated and the down-converted signal had a good eye diagram compared with the original one.

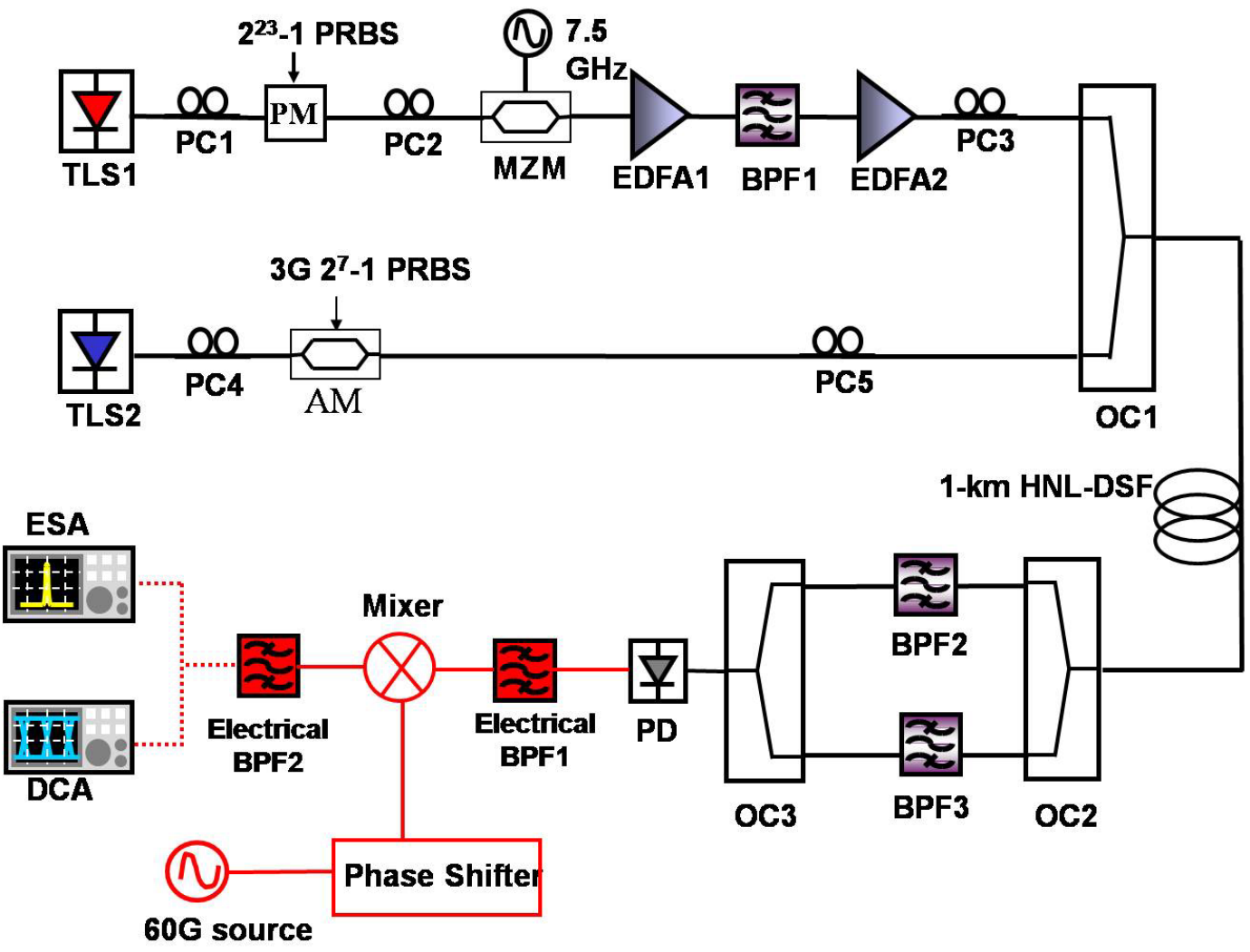

Fig. 7 Experimental setup for simulation of $60-\mathrm{GHz}$ frequency up-conversion.
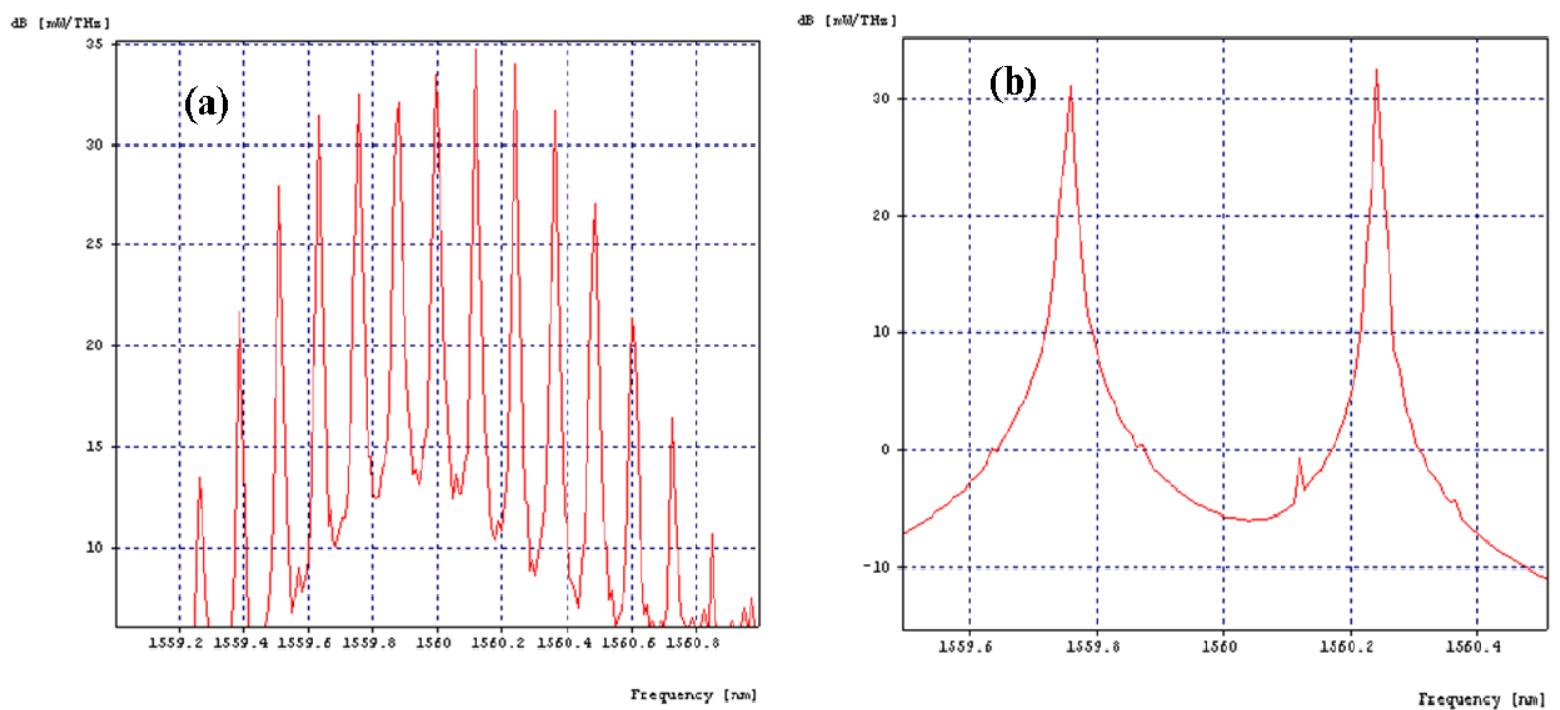

Fig. 8 (a) Optical spectrum of the signal after HNL-DSF. (b) Optical spectrum of the two second-order sidebands after filtering. 

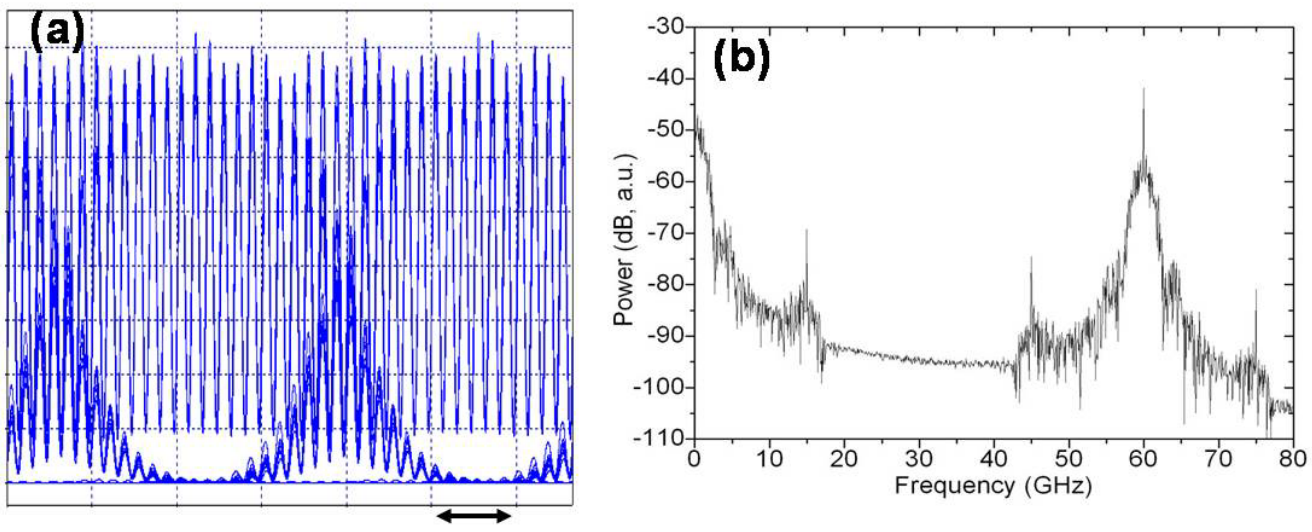

$100 \mathrm{ps} / \mathrm{div}$
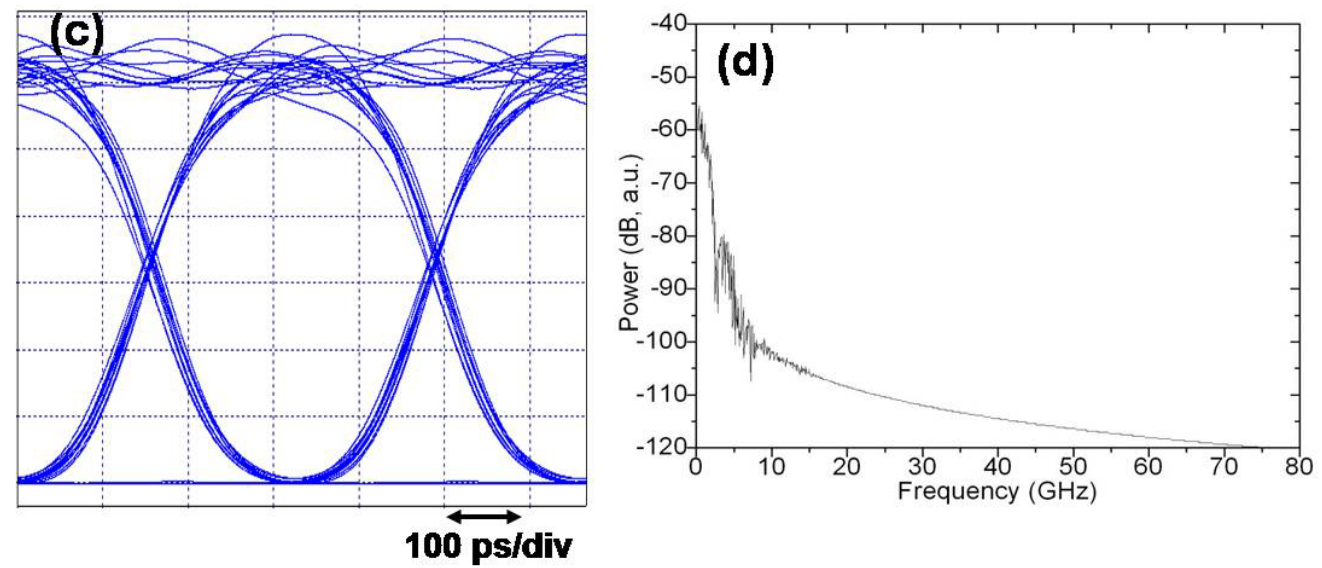

Fig. 9 (a,b) Waveform of up-converted 60-GHz signal and its corresponding power spectrum. (c,d) Electrical down-converted baseband signal and its corresponding power spectrum.

\section{CONCLUSION}

A novel approach for 19-GHz UWB signal generation is proposed and demonstrated in the experiment. By using highrepetition pulsed-pump fiber OPA, optical spectrum of the amplified signal consists of many discrete frequency peaks separated by the modulation frequency of the pump, and each frequency peak contains the same spectral information as the original signal. After selecting the central two or three main frequency components and beating in the PD, a 19-GHz UWB signal is generated. In addition, this approach can be extended into a $60-\mathrm{GHz}$ up-conversion case without using electrical source or electrooptic modulators with a higher speed. It can be achieved by simply selecting two second-order sidebands and beating in the PD. Simulation results show that the 3-Gb/s PRBS signal is successfully up-converted into $60-\mathrm{GHz}$ band and the down-converted signal has a good waveform compared with the original one. This method has the advantages of high-speed operation because of the ultra-fast response time of fiber OPA, and can be upgraded into 60GHz up-conversion system.

\section{ACKNOWLEDGMENT}

The work described in this paper was partially supported by grants from the Research Grants Council of the Hong Kong Special Administrative Region, China (Projects No. HKU 7172/07E and HKU 7179/08E). 


\section{REFERENCES}

1. J. P. Yao, F. Zeng, and Q. Wang, "Photonic generation of ultrawideband signals," J. Lightwav. Technol., 25(11), 3219-3235 (2007).

2. Q. Wang, F. Zeng, S. Blais, and J. Yao, "Optical ultrawideband monocycle pulse generation based on cross-gain modulation in a semiconductor optical amplifier," Opt. Lett., 31(21), 3083-3085 (2006).

3. J. Li, B. P. P. Kuo, and K. K. Y. Wong, "Ultra-wideband pulse generation based on cross-gain modulation in fiber optical parametric amplifier," IEEE Photon. Technol. Lett., 21(4), 212-214 (2009).

4. F. Zeng and J. P. Yao, "Ultrawideband impulse radio signal generation using a high-speed electrooptic phase modulator and a fiber-Bragg-grating-based frequency discriminator," IEEE Photon. Technol. Lett., 18(19), 2062-2064 (2006).

5. I. S. Lin, J. D. McKinney, and A. M. Weiner, "Photonic synthesis of broadband microwave arbitrary waveforms applicable to ultra-wide-band communication," IEEE Microw. Wireless Compon. Lett., 15(4), 226-228 (2005).

6. X. Qian, P. Hartmann, A. Wonfor, J. D. Ingham, V. Penty, I. H. White, "Microwave signal transmission over a directly-modulated radio-over-fiber link using cascaded semiconductor optical amplifiers," in Proc. Opt. Fiber Commun. Conf., 2005, OThB2.

7. G. H. Smith, D. Novak, and Z. Ahmed, "Overcoming chromatic-dispersion effects in fiber-wireless systems incorporating external modulators," IEEE Trans. Microw. Theory Tech., 45(8), 1410-1415 (1997).

8. R. Hofstette, H. Schmuch, R. Heidemann, "Dispersion effects in optical millimeter-wave systems using selfheterodyne method for transport and generation," IEEE Trans. Microw. Theory Tech., 43 (9), 2263-2269 (1995).

9. J. Yu, J. Gu, X. Liu, Z. Jia, and G. K. Chang, "Seamless integration of an 8 x $2.5 \mathrm{~Gb} / \mathrm{s} \mathrm{WDM-PON}$ and radio-overfiber using all-optical up-conversion based on raman-assisted FWM," IEEE Photon. Technol. Lett., 17(9), 1986-1988 (2005).

10. H. J. Song, J. S. Lee, and J. I. Song, "Signal up-conversion by using a cross-phase-modulation in all-optical SOAMZI wavelength converter," IEEE Photon. Technol. Lett., 16(2), 593-595 (2004).

11. Y. K. Seo, C. S. Choi, and W. Y. Choi, "All-optical signal up-conversion for radio-on-fiber applications using crossgain modulation in semiconductor optical amplifiers," IEEE Photon. Technol. Lett., 14(10), 1448-1450 (2002).

12. J. Yu, Z. Jia, L. Yi, Y. Su, G. K. Chang, and T. Wang, "Optical millimeter-wave generation or up-conversion using external modulators," IEEE Photon. Technol. Lett., 18(1), 265-267 (2006).

13. T. Kuri, Y. Omiya, T. Kawanishi, S. Hara, and K. Kitayama, "Optical transmitter and receiver of 24-GHz ultrawideband signal by direct photonic conversion technique," in Int. Topical Meeting Microwave Photonics, Grenoble, France, Oct. 2006.

14. Y. L. Guennec and R. Gary, "Optical frequency conversion for millimeter-wave ultra-wideband-over fiber systems," IEEE Photon. Technol. Lett., 19(13), 996-998 (2007).

15. S. Fu, W. Zhong, Y. J. Wen, and P. Shum, "Photonic monocycle pulse frequency up-conversion for ultrawidebandover-fiber applications," IEEE Photon. Technol. Lett., 20(12), 1006-1008 (2008).

16. Q. Chang, Y. Tian, T. Ye, J. Gao, and Y. Su, "A 24-GHz ultra-wideband over fiber systems using photonic generation and frequency up-conversion," IEEE Photon. Technol. Lett., 20(19), 1651-1653 (2008).

17. H. C. Chien, A. Chowdhury, Z. Jia, Y. T. Hsueh, and G. K. Chang, " $60 \mathrm{GHz}$ millimeter-wave gigabit wireless services over long-reach passive optical network using remote signal regeneration and upconversion," Opt. Express, 17(5), 3036-3041 (2009).

18. G. Kalogerakis, M. E. Marhic, and L. G. Kazovsky, "Multiple-wavelength conversion with gain by a highrepetition-rate pulsed-pump fiber OPA,” J. Lightw. Technol., 23(10), 2954-2960 (2005). 